\title{
Individual Fibre Inclination Segmentation from X-ray Computed Tomography using Principal Component Analysis
}

Salling, Filip Bo; Jeppesen, Niels; Sonne, Mads Rostgaard; Hattel, Jesper H.; Mikkelsen, Lars Pilgaard

Published in:

Journal of Composite Materials

Link to article, DOI:

$10.1177 / 00219983211052741$

Publication date:

2022

Document Version

Peer reviewed version

Link back to DTU Orbit

Citation (APA):

Salling, F. B., Jeppesen, N., Sonne, M. R., Hattel, J. H., \& Mikkelsen, L. P. (2022). Individual Fibre Inclination Segmentation from X-ray Computed Tomography using Principal Component Analysis. Journal of Composite Materials, 56(1), 83-98. https://doi.org/10.1177/00219983211052741

\section{General rights}

Copyright and moral rights for the publications made accessible in the public portal are retained by the authors and/or other copyright owners and it is a condition of accessing publications that users recognise and abide by the legal requirements associated with these rights.

- Users may download and print one copy of any publication from the public portal for the purpose of private study or research.

- You may not further distribute the material or use it for any profit-making activity or commercial gain

- You may freely distribute the URL identifying the publication in the public portal 


\title{
Individual Fibre Inclination Segmentation from X-ray
}

\section{Computed Tomography using Principal Component Analysis}

\author{
Filip Bo Salling ${ }^{\mathrm{a} 1}$, Niels Jeppesen ${ }^{\mathrm{b}}$, Mads Rostgaard Sonne ${ }^{\mathrm{a}}$, Jesper H. Hattel ${ }^{\mathrm{a}}$ and \\ Lars Pilgaard Mikkelsen ${ }^{\mathrm{c} 2}$ \\ ${ }^{a}$ Department of Mechanical Engineering, Technical University of Denmark (DTU), \\ 2800 Kgs. Lyngby, Denmark. \\ ${ }^{\mathrm{b}}$ Department of Applied Mathematics and Computer Science, DTU, Denmark. \\ ${ }^{\mathrm{c}}$ Department of Wind Energy, DTU, Denmark.
}

Corresponding authors: ${ }^{1} \underline{\text { fsras@mek.dtu.dk, }}{ }^{2} \underline{\text { lapm@dtu.dk }}$

\begin{abstract}
This study presents a holistic segmentation procedure, which can be used to obtain individual fibre inclination angles from X-ray computed tomography. The segmentation approach is based on principal component analysis and was successfully applied for a unidirectional and an air textured glass fibre reinforced composite profile. The inclination results show a weighted mean fibre inclination of $2.1^{\circ}$ and $8.0^{\circ}$ for the unidirectional and air textured profile, respectively. For the air textured composite, fibre inclinations of up to $55^{\circ}$ were successfully segmented. The results were verified by comparative analysis with equivalent results obtained from structure tensor analysis showing no notable deviation. The comparable characteristics in combination with the distinct differences of the two material systems make this case study ideal for verification and validation of idealized models. It is shown how this approach can provide fast, accurate and repeatable inclination estimates with a high degree of automation.
\end{abstract}


Keywords: Glass fibre composites, Fibre undulation, Segmentation verification, Singular value decomposition, Structure tensor.

\section{Introduction}

The demand for lightweight materials in the form of fibre reinforced polymer (FRP) composites has consistently increased and the growth is expected to continue [1]. FRP composite materials are heavily used for structural applications where high specific mechanical properties are required, e.g., high stiffness to weight ratio. In that regard, the FRPs can easily compete with most metal alloys and are therefore heavily used in offshore-, construction-, and wind turbine industries [2-3]. This is one of the reasons why the growth rate of composites usage has exceeded that of steel and aluminium in the last decades [4].

The macroscopic properties of FRPs are inherently related to their microstructure, which is mainly described by the fibre volume fraction and the fibre architecture. This enables tailoring of mechanical properties for a given application. Hence, numerous studies investigate the relation between composite microstructure and macroscopic properties, e.g., stiffness, compressive strength and fatigue properties.

In the study by Paluch [5], the effect of fibre undulation in unidirectional (UD) composite was investigated by destructive characterization, i.e., regularly cut sections examined by optical microscopy. It was concluded that considering a composite material as a series of mutually parallel fibres undulating in phase is a poor assumption. Non-destructive confocal laser scanning microscopy was used in [6] to investigate fibre undulation. However, this method is limited to sufficient transparent samples, i.e., composites where the matrix fluoresces strongly and with fibre volume fraction below 30 percent.

X-ray Computed Tomography (XCT) is widely used to characterize material properties of fibre reinforced polymers, as well as for materials like concrete and metals [7-9]. XCT has the advantage of nondestructive testing and a high resolution, i.e., micron level voxel size [10-11]. However, the resolution obtained by XCT cannot compete with that obtained by scanning electron microscopy (SEM), reaching sub-micron resolutions [12]. XCT was used to investigate the effect of varying off-axis angles in non-crimp fabrics [13-14].

In a recent study [15], the effect of fibre misalignment on the longitudinal compressive and tensile failure was investigated by micromechanical modelling, introducing the fibre misalignment using a stochastic process. Similar mechanical modelling was conducted on characterized microstructures by 
Auenhammer et al. [16]. The main objective of this study was to develop a highly automated process to transfer XCT data into robust finite element models. The model was evaluated by its ability to predict Young's moduli of UD fibre composites loaded in tension and it was concluded that the methodology did not suffice to predict the correct trend. It was concluded that the reason for the deviations most likely was attributed to the single bundle fibre tracking segmentation process and in particular the mean fibre orientation, fibre volume fraction, and bundle to volume ratio. The influence of the segmentation method was investigated in [17] and the waviness induced by backing bundles was captured by generating a boundary between the backing and UD bundles. The approach in [16-17] focusses on the bundle level rather than the fibre level, hence, the influence of the single fibre scale was not investigated. It is argued that the advantage of the methodology is automation, but it is also mentioned that the user needs experience in Avizo to set up the right parameters depending on the composite architecture and the fibre to matrix attenuation contrast.

The structure tensor (ST) segmentation method was used by Advani and Tucker to predict fibre orientations in short fibre composites [18]. The ST method was recently used to characterize a non-crimp glass fibre fabric and the Jupyter notebooks and Python code behind the segmentation process were publicly shared [19]. Another open-source Python package is available, i.e., 'Quanfima' which successfully applies the ST approach to 3D biomaterial datasets [20]. This ST segmentation approach uses thresholding to separate fibre and matrix material, which necessitates a proper attenuation contrast as well as spatially separable fibres.

Emerson et al. [21] developed an interactive fibre tracking algorithm, which was used to characterize a UD composite. The algorithm was developed in MATLAB, publicly shared, and statistical validated in [10]. Its applicability for a textured fibre composite was investigated in [22]. It was found that the algorithm made some erroneous fibre detections for high angle fibres, but it was in general applicable for textured fibre composites.

In a study by Amrehn et al. [23], it was concluded that interactive segmentation methods are beneficial for complex structures as opposed to fully automated segmentation methods that inherently lack domain knowledge. 


\subsection{Objective}

FRP composites are often designed with large safety margins because of incomplete knowledge about the connection between the microstructure and the macroscopic properties.

The main objective of this study is to use XCT to quantify the fibre undulation of two mutually distinct composite systems. The first one is a pultruded UD glass fibre profile and the second one is a pultruded air textured glass fibre profile. The air textured fibre reinforced composite is expected to have a complex structure, which calls for an interactive segmentation method. The segmentation will build upon the individual fibre tracking algorithm developed in [21]. The inclination of the fibres will be analysed using a novel segmentation method developed by the authors of the present work. The new segmentation approach will include a semi-automatic segmentation module based on principal component analysis (PCA). The quantified inclination results will be verified by comparison with corresponding results obtained by the ST method, as described in [19]. Finally, the prospects of novel insight into the connection between microstructure and macroscopic properties will be discussed.

\section{Materials}

This study is carried out on two mutually distinct FRP composite systems, namely a UD glass fibre reinforced thermoset composite (UD-fib) and an air textured glass fibre reinforced thermoset composite (air-tex). The two composites are both manufactured by the resin injection pultrusion process using solely fibre bundle rovings. Hence, the fibre architecture is inherently continuous through the profile and any observable fibre inclinations are thus not a result of misaligned layup, which sometimes is the case in prepreg and non-crimp fabric composites [24].

The UD-fib and the air-tex composites are manufactured as a rod with a diameter of $\sim 13 \mathrm{~mm}$ and a large beam with a square cross-section of $\sim 100 \times 100 \mathrm{~mm}^{2}$, respectively. But, for the purpose of the current study, both pultruded samples were machined to obtain a rod with a diameter of $\sim 5 \mathrm{~mm}$, see Figure 1 . The continuous UD- and air textured glass fibre rovings used for the two composites are of the same glass fibre type with an estimated average diameter of $\sim 23.4 \mu$ m, i.e., the air textured rovings are a textured version of the UD rovings. This makes the two composite systems suitable for comparative analysis. The two composite rods together with a schematic illustration of their respective fibre reinforcement are depicted in Figure 1. 


\section{FIGURE 1}

It should be noticed how Figure 1 is set up with a) UD-fib and b) air-tex. This figure setup is continued throughout the paper to ease comparative analysis and is additionally directly comparable with the corresponding a) and b) figures in the data article published by the authors [12].

\section{X-ray Computed Tomography}

To recap, the scope of the current study is to characterize the fibre orientation of the two mutually distinct FRP composite systems, i.e., the UD-fib and air-tex material described in the previous section. This is done using XCT followed by an advanced novel segmentation approach. In the following section the experimental XCT method is described.

\subsection{Experimental Method (XCT)}

The XCT experiments were carried out on a Zeiss Xradia Versa 520 scanner with a voltage of $40 \mathrm{kV}$. The scan was performed with $4 \times$ optical magnification obtaining 4501 projections at binning 2 . A tomographic volume of $988 \times 1013 \times 999$ voxels with a voxel size of $1.99 \mu \mathrm{m}$ was obtained. The tomographic data is published in the data article [12], including a detailed description of the X-ray tomography settings. The same procedure was used for the two composite systems and the resulting 3D tomograms are depicted together with an example of a tomographic cross-sectional slice in Figure 2.

\section{FIGURE 2}

The tomographic reconstruction shown in Figure 2 has a high resolution, i.e., a voxel size of $1.99 \mu \mathrm{m}$. It also has a high attenuation contrast, which eases the separation of the constituent fibre and matrix materials by the naked eye. However, obtaining a segmented mathematical description of the individual fibres is not a trivial task, as the fibres are often bundled closely together, making separation difficult, even with high attenuation contrast data. A novel segmentation process is described in the following section.

\section{Individual Fibre Segmentation}

In the current study the segmentation process is divided into three steps: i) a slice-by-slice fibre centre detection, ii) a fibre tracking procedure relating centres of the individual image slices to each other to form continuous fibres, and iii) a fibre inclination segmentation step, i.e. assigning an inclination angle to each 
individual fibre trajectory. The first two segmentation steps were conducted using the MATLAB algorithm developed by Emerson et al. in [21]. The third and final segmentation step was performed using a novel approach developed in this study. The three segmentation steps are the subject of the following 3 sections. The segmentation process was continuously evaluated for both material systems to ensure a generic segmentation process applicable for both material systems. This section is concluded with a summary including a table with an overview of the user input and running time for each of the three segmentation process steps (see Table 2).

\subsection{Fibre centre detection}

The first step of the segmentation process, i.e., the slice-by-slice fibre centre detection, was conducted using the algorithm developed my Emerson et al [21]. This segmentation algorithm uses a training step to set up a dictionary of corresponding image and label patches. The dictionary was set up using the following steps:

i) Per default, the middle slice of the tomographic stack of slices was chosen for the training step, i.e., slice no. 500 out of the 999 slices

ii) The region of interest (RoI) was chosen to include the entire tomographic volume.

iii) An image region for the training step was chosen somewhat smaller than the RoI but still covering a major part of a tomographic slice.

iv) An optimal patch size of 11 pixels, corresponding to approximately 1 fibre diameter, was chosen based on the voxel size of $1.99 \mu \mathrm{m}$.

v) Finally, the graphical user interface was used to setup the dictionary.

The accuracy of the fibre centre detection algorithm was evaluated qualitatively by manual inspection of individual tomographic slices and the corresponding fibre centres detected. Figure 3 illustrates an example of a tomographic slice and the corresponding detected fibre centres. It should be noticed how the dictionary successfully detects the fibre centres with very few exceptions. The erroneous double detections and missing fibre detections make up less than $0.1 \%$ of total number of fibres and are therefore considered insignificant for the overall evaluation of the microstructure and hence the scope of this study (see Figure 3).

\section{FIGURE 3}

The dictionary-based fibre centre detection algorithm was applied for the stack of 999 images resulting in a processing time of approximately one hour for each of the two tomographic volumes. The total 3D 
volume of detected fibre centres are depicted by four evenly spaced stacks of five image slices, see Figure 4.

\section{FIGURE 4}

The fibre centres shown in Figure 4 clearly illustrates a pattern of continuous fibres for both material systems. By comparison of Figure 2 and 4 it should be noticed how the detected fibre centres indicate the expected difference between the two material systems.

At the top and bottom of the tomographic stack of image slices significantly increased noise was observed. The noise arises from the well-known cone beam effect and results in missing fibre detections. However, missing fibre detections in one image has no influence on the fibre detections in the other images. It does however influence the second step of the segmentation process, i.e., the individual fibre tracking. This problem is accounted for by excluding the top and bottom 50 image slices. This will be discussed in the following section about the fibre tracking segmentation step.

\subsection{Fibre tracking}

When the fibre centre detection has been conducted for the full stack of tomographic image slices (cf. Figures 3 and 4), the next step is to track the 2D slices of fibre centres through the depth of the volume, i.e., along the x-axis. Hence, connecting fibre centres belonging to the same fibre and thereby obtaining the individual fibre trajectories. This segmentation step is also performed using the algorithm by Emerson et al. [21]. The only user input for the fibre tracking algorithm is how many pixels a fibre centre is allowed to move from one slice to the next, i.e., a $2 \mathrm{D}$ planar distance. Thus, this is naturally a function of the voxel size and the fibre diameter. From simple geometrical considerations an allowable fibre centre movement from one slice to the next of 6 pixels will allow for a maximum inclination angle of approximately $80^{\circ}$. Hence, an allowable movement of 6 pixels was chosen as a conservative input (cf. Figure 2).

The fibre tracking algorithm is set up so the number of fibre centres detected in the very first image slice dictates how many fibres in total the tracking algorithm will allow. Thus, the less accurate fibre centre detection at the very top or bottom image slices, results in too few fibre trajectories. Therefore, it was chosen to ignore the top and bottom 50 image slices. This solved the above-mentioned problem at the expense of a decreased tomographic volume. 
The individual fibre tracking algorithm was applied for both material systems, each with a processing time of less than five minutes. The 3D volume of all fibre trajectories obtained from the fibre tracking algorithm are shown in Figure 5.

\section{FIGURE 5}

A 3D volume representing 10 percent of the fibre trajectories is depicted in Figure 6.

\section{FIGURE 6}

Figures 5 and 6 show the expected differences of the fibre trajectories between the less and more misaligned material cases (cf. Figure 2).

However, taking a closer look at Figure 6(b) one can notice a few fibre trajectories with piecewise unlikely geometries, i.e., UD fibre kink segments along the edge of the tomographic volume. This is most likely due to the fact that the fibre tracking algorithm does not allow termination or initiation of fibre trajectories running in or out of the tomographic volume. Hence, two individual fibres might get connected by a UD fibre segment to form a single fibre connected by this "fictive" UD fibre kink. Quantification of this type of error would involve visual inspection. This was manually done by the authors and the frequency of this error was judged to have no significant influence on the scope of this study, i.e., characterizing the fibre undulation. Hence, using the fibre trajectories obtained it is now possible to evaluate and quantify the inclination of the individual fibres.

\subsection{Fibre inclination estimation using PCA}

The third and final step of the segmentation process is inclination estimation of the individual fibre trajectories. The segmentation algorithm, used for fibre detection and fibre tracking, does have a third module for orientation estimation which was successfully used for UD fibre composites [10,20]. However, the module assumes a straight fibre trajectory between the fibre centres at the top and bottom image slice and does not take any undulation into account. From Figure 6 it is evident that the air textured fibres in the air-tex sample exhibit significant undulation, which should be resolved. To do so, a novel inclination segmentation algorithm was developed. The idea behind the current approach is to evaluate the inclination of each individual fibre as a weighted mean of fibre sub-segments, e.g., each individual fibre is divided into a number of sub-segments by dividing the total stack of image slices into smaller stacks. For instance, the stack of 900 image slices is divided into two stacks of 450 image slices, hence, each fibre is represented by two fibre sub-segments (see Figure 7). 


\section{FIGURE 7}

The straight lines representing the fibre sub-segments (cf. Figure 7) are obtained from linear regression analysis using Principal Component Analysis (PCA) [25].

PCA is a statistical interpretation of the singular value decomposition (SVD) of a data matrix $\boldsymbol{A}$. Once the matrix $\boldsymbol{A}$ is set up, the SVD is done in MATLAB. The results of the SVD are the matrices $\boldsymbol{U}, \boldsymbol{\Sigma}$ and $\boldsymbol{V}^{\boldsymbol{T}}$. The covariance matrix $\boldsymbol{U}$ contains the left singular vectors, $\boldsymbol{\Sigma}$ is a diagonal matrix with the singular values ordered by importance and $\boldsymbol{V}^{T}$ is a transposed matrix containing the right singular vectors. Hence, the SVD yields

$$
A=U \Sigma V^{T}
$$

The steps of the regression analysis are: i) Setup a matrix $\boldsymbol{X}$ with all fibre centre coordinates from the fibre segment of interest. ii) Compute the mean fibre centre coordinate $\overline{\boldsymbol{X}}$, i.e., the centre point of the regression line of interest. iii) Compute the matrix $\boldsymbol{A}=\boldsymbol{X}-\overline{\boldsymbol{X}}$. iv) Compute the covariance matrix $\boldsymbol{U}$ by singular value decomposition of $\boldsymbol{A}$. The first left singular vector $\boldsymbol{u}_{\mathbf{1}}$ of the covariance matrix $\boldsymbol{U}$, corresponding to the highest singular value $\sigma_{1}$, is the first principal component and gives the direction of the best fit line. Hence, the regression line, representing the fibre sub-segment of interest, goes through the mean centre point $\overline{\boldsymbol{X}}$ and has the direction $\boldsymbol{u}_{1}$. v) Compute the coordinates of the best fit line using the parametric vector equation of a line in three dimensions given by

$$
\boldsymbol{X}_{\text {fit }}=\overline{\boldsymbol{X}}+t \cdot \boldsymbol{u}_{1}
$$

where, $\boldsymbol{X}_{\boldsymbol{f i t}}$ is the resulting coordinates of the best fit line and $t$ is a scaling factor.

Finally, the increments $\Delta x, \Delta y$ and $\Delta \mathrm{z}$ are readily obtained from the endpoint coordinates of $\boldsymbol{X}_{f i t}$. The inclination of the linear fibre sub-segments is then computed using simple trigonometry. The inclination angle, i.e., the angle with respect to the $\mathrm{x}$-axis, is calculated as

$$
\theta_{x}=\arctan \left(\frac{\sqrt{\Delta y^{2}+\Delta z^{2}}}{\Delta x}\right)
$$

The individual fibre inclination is weighted by their corresponding fibre length. Thus, the fibre length of each fibre sub-segment is also calculated. The length of each fibre segment is given as

$$
L_{f}=\sqrt{\Delta x^{2}+\Delta y^{2}+\Delta z^{2}}
$$


The alignment of the samples prior to the X-ray scan can be evaluated by projected inclinations and these are therefore also computed. The projected inclination angles and corresponding projected 'fibre lengths' are calculated using the simplified 2D version of equations (3) and (4). The equations for the xyprojections are

$$
\begin{aligned}
& \theta_{x y}=\arctan \left(\frac{\Delta y}{\Delta x}\right) \\
& L_{f}^{x y}=\sqrt{\Delta x^{2}+\Delta y^{2}}
\end{aligned}
$$

and the equivalent equations for the xz-projection are (5) and (6) with z substituted for $y$.

4.3.1. Segmentation discretization. An example of a fibre trajectory and the corresponding representation by five linear sub-segments are illustrated in Figure 8.

\section{FIGURE 8}

Figure 8 shows that the linear segments, obtained from the regression analysis, represents the undulation of the individual fibre well, i.e., each straight sub-segment follows the local fibre trajectories, while imposing a piecewise smoothing effect to the trajectory. This is beneficial because it minimizes the effect of the noise, which arises from the uncertainty of the fibre centre detections. Hence, there is a trade-off between resolving the fibre undulation and maintaining the smoothening effect, i.e., the uncertainty related to the fluctuating fibre centre detections should not be resolved (cf. Figure 8(a)). Therefore, a proper discretization of the fibre trajectories must be chosen to obtain good results. The fibre trajectories are now discretized into fibre sub-segments with a fixed height $\Delta x$ corresponding to an integer number of image slices. For instance, each of the five fibre sub-segments in Figure 8 contains fibre centres of (up to) 180 image slices corresponding to a fibre sub-segment height of approximately $\Delta x=358 \mu \mathrm{m}$.

For the sake of generalization, the segmentation mesh will be described using the normalized mesh size $\Delta \mathrm{x} / D_{f}$, using an average fibre diameter of $D_{f}=23.4 \mu \mathrm{m}$, confer section 2 . Which, in the case with five fibre sub-segments illustrated in Figure 8 corresponds to a normalized segmentation mesh size of $\Delta \mathrm{x} / D_{f} \approx$ 15. The method was tested by varying the normalized mesh size from 77 (i.e., including all 900 tomographic image slices to represent the fibre trajectory as a single straight fibre segment, cf. Figure 7) to 1 (i.e., representing the fibre trajectories by 81 straight fibre sub-segments each obtained by 12 tomographic image 
slices). Figure 9 illustrates a fibre trajectory and the corresponding representation by straight fibre subsegments obtained using the fine segmentation mesh where $\Delta \mathrm{x} / D_{f} \approx 1$.

\section{FIGURE 9}

FIGURE 10

Figure 9 shows that a fine segmentation mesh captures some of the noise related to the uncertainty of the fibre centre detections. This indicates that a mesh size of $\Delta \mathrm{x} / D_{f} \approx 1$ does not provide sufficient smoothing. The goal is to obtain a good resolution of the fibre undulation while maintaining the smoothing effect from the regression analysis. A dimensionless segmentation mesh size of $\Delta \mathrm{x} / D_{f} \approx 10$, which corresponds to including 113 image slices for each fibre sub-segment, provides a good trade-off for the current data. The choice of $\Delta \mathrm{x} / D_{f} \approx 10$ is based on the known voxel size of $1.99 \mu \mathrm{m}$, an expected fibre diameter of $\sim 23.4 \mu \mathrm{m}$ as well as considerations regarding the physical limitation of the frequency of the fibre undulation. Figure 10 shows a well-defined fibre trajectory from the air-tex sample using the dimensionless segmentation mesh size of $\Delta \mathrm{x} / D_{f} \approx 10$. An overview of the differences between different dimensionless segmentation mesh sizes is summarized in table 1.

\section{TABLE 1}

Each linear fibre sub-segment was only accepted for PCA if it contains at least $95 \%$ of the possible fibre centres. This conservative criterion was chosen to minimize any artificial inclination effects from erroneous fibre detection and fibre tracking.

The PCA based individual fibre inclination segmentation algorithm was applied for both materials systems using a normalized segmentation mesh size of $\Delta \mathrm{x} / D_{f} \approx 10$, resulting in a processing time for each of the material systems of less than 15 seconds.

\subsection{Individual fibre segmentation summary}

A schematic overview of the three-step segmentation method used in the present study is summarized in Table 2 .

\section{TABLE 2}

The three segmentation steps consist of: i) Fibre centre detection, ii) Fibre tracking and iii) Inclination estimation. The first two steps are performed using the fibre tracking algorithm [21], while a novel segmentation algorithm, using PCA, was developed for inclination estimation in this study. The user inputs for the three step segmentation modules are summarized in Table 2, together with the running time for each of the three segmentation steps (using a standard laptop equipped with an Intel ${ }^{\circledR}$ Core ${ }^{\mathrm{TM}}$ i5-6300U CPU, 
Intel ${ }^{\circledR}$ HD Graphics 520 and $8 \mathrm{~GB}$ of RAM). The total processing time of the complete segmentation procedure is only a little more than one hour.

\section{Results}

The PCA algorithm was used to obtain the fibre inclination distribution for both material systems. The results are presented in section 5.2, but first the choice of segmentation mesh is evaluated in the following section.

\subsection{Segmentation mesh}

The choice of $\Delta \mathrm{x} / D_{f}=10$ as an 'optimal' dimensionless segmentation mesh size (cf. section 4) was determined by varying the dimensionless segmentation mesh size from 77 (representing each fibre using a single straight line) to a value of 1 (representing each fibre by 81 sub-segment straight lines). The influence of the variation in segmentation mesh on the overall inclination estimation was evaluated by comparing the weighted mean inclination for the total volume, calculated as

$$
\bar{\theta}_{x}=\sum_{i=1}^{N_{s}}\left(\theta_{i} \cdot \frac{L_{f, i}}{L_{f}^{t o t}}\right)
$$

where the summation index $i$ is used to sum over the total number of fibre sub-segments $N_{s}$ and $L_{f}^{\text {tot }}$ is the total fibre length of the segmented 3D volume, given by the sum of all the fibre sub-segments

$$
L_{f}^{t o t}=\sum_{i=1}^{N_{s}} L_{f, i}
$$

The weighted mean inclination as a function of the dimensionless segmentation mesh size is depicted in Figure 11.

\section{FIGURE 11}

Figure 11 shows how the refinement of the segmentation mesh from $\Delta \mathrm{x} / D_{f}=77$ to $\Delta \mathrm{x} / D_{f}=10$ results in an increase in the mean fibre inclination of approximately $15 \%$ and $20 \%$ for the UD-fib and air-tex material systems, respectively. Hence, the segmentation mesh has a significant influence on the inclination estimation for both material systems, i.e., the fibre undulation is resolved. It should further be noticed how changes in $\Delta \mathrm{x} / D_{f}$ around 10 has no significant influence on the mean inclination, i.e., $<0.05$ (cf. Figure 11). 
The mean inclination increases significantly at very fine mesh sizes, in particular when $\Delta \mathrm{x} / D_{f}=1$. This increase highlights the importance of reducing the impact of the noise, by using more slices for each segment (cf. Figure 9). This is particularly evident for the UD-fib sample, where the deviations in the centre detections cause a significant relative increase in the mean fibre inclination when $\Delta \mathrm{x} / D_{f}$ is low. This is expected because the noise becomes significant due to the low inclination angles inherently expected from a UD fibre reinforced composite.

Hence, a value of $\Delta \mathrm{x} / D_{f}=10$ was found to provide a good trade-off between resolving fibre undulation and maintaining the smoothing effect of the analysis and is therefore used in the analysis henceforth.

\subsection{Inclination distribution}

The weighted inclination distribution obtained using a segmentation mesh size of $\Delta \mathrm{x} / D_{f}=10$ is illustrated in Figure 12.

\section{FIGURE 12}

The inclination distributions depicted in Figure 12 consist of the actual inclination distribution (top) and the projected inclination distributions Figure 12 (middle and bottom). The inclination distributions in Figure 12 (top) are right-angled distributions, which inherently arises from the nature of the fibre reinforcement, i.e., continuous fibre bundles. The main characteristics of the inclination distributions illustrated in Figure 12 are summarized in Table 3, i.e., the weighted mean and the quartiles of the inclination distribution as well as the weighted mean of the projected inclination distributions.

\section{TABLE 3}

The results illustrated and summarized in Figure 12 and Table 3 show expected magnitudes of inclination angles compared with the corresponding 3D tomograms depicted in Figure 2. From Table 3 it should be noticed how $\bar{\theta}_{x}>\bar{\theta}_{x y}$ and $\bar{\theta}_{x}>\bar{\theta}_{x z}$, which indicate little tilting of the samples in the XCT setup. Hence, the inclination estimation is qualitatively acceptable. The fibre trajectories are plotted with colours corresponding to their individual mean fibre inclination using the quartiles as interval boundaries (see Figure 13).

\section{FIGURE 13}

The fibre trajectories coloured according to their inclination angle (see Figure 13) show that the PCAbased segmentation algorithm estimates the inclination of the individual fibres well. A cross-sectional view of the coloured fibre trajectories is illustrated in Figure 14. 


\section{FIGURE 14}

The cross-sectional view in Figure 14 shows how fibres with similar inclination angles tend to group in bundle like structures. A study of these trends could be of interest for future studies.

The estimated inclinations can be used to evaluate mechanical properties, e.g., estimation of longitudinal stiffness using the fibre efficiency formula proposed by Krenchel [26]. The microstructural effect on mechanical properties is the subject of ongoing studies by the authors. The preliminary results indicate that the inclination estimations obtained in this study can be used to explain some of the deviation in longitudinal stiffness observed comparing tensile test results with classical rule of mixture estimates. Hence, this would be of great interest in future studies.

For verification, the results of the PCA segmentation method were compared to equivalent fibre inclination estimates obtained using the structure tensor method - this is the subject of section 6 .

\section{Verification}

Microstructure investigations using XCT and subsequent numerical segmentation will inherently contain errors since the segmented tomogram is a discretized mathematical representation of the microstructure features. Hence, verification is necessary to evaluate the error of this mathematical segmentation method. The developed mathematical method using PCA is verified by comparison with equivalent results obtained for the same XCT scan using the structure tensor (ST) method.

\subsection{The structure tensor method}

The ST method, when applied on volumetric data, can be used to extract local structural information from the data, such as local orientations of fibre-like structures. The structure tensor itself is a 3-by-3 matrix, summarizing local gradient information around a point in space. One way to calculate the structure tensor is using a Gaussian derivative for computing the gradient and a Gaussian window for integration [19]. The size of the Gaussian derivative kernel is determined by the parameter $\sigma$, while the size of the Gaussian integration kernel is determined by the parameter $\rho$. The parameters $\sigma$ and $\rho$ are also known as the noise scale and integration scale, respectively. Formally, the structure tensor $\mathbf{S}$ can be formulated as

$$
\mathbf{S}=K_{\rho} \cdot\left(\nabla V_{\sigma}\left(\nabla V_{\sigma}\right)^{T}\right)
$$


where $\nabla V_{\sigma}$ is the gradient computed using the Gaussian derivative kernel that depends on $\sigma$ and $K_{\rho}$ is the Gaussian integration kernel, which depends on $\rho$.

Once $\mathbf{S}$ for a point has been computed, the predominant orientation can be determined through eigendecomposition of $\mathbf{S}$. The result is three positive eigenvalues $\lambda_{1} \leq \lambda_{2} \leq \lambda_{3}$ and their corresponding mutually orthogonal eigenvectors $\mathbf{v}_{1}, \mathbf{v}_{2}$, and $\mathbf{v}_{3}$. The direction of least variation is the one of $\mathbf{v}_{1}$ corresponding to the smallest eigenvalue $\lambda_{1}$.

The structure-tensor Python package [19] is used to compute $\mathbf{S}$ for each voxel in the tomographic volume. The parameters used are $\sigma=1.045$ and $\rho=4.18$, which were chosen based on the voxel resolution and fibre diameter. After this, the dominant orientation, $\mathbf{v}_{1}$, is calculated using the same Python package. The structure tensor $\mathbf{S}$ is computed for each voxel position. However, the goal is to estimate the fibre orientation distribution, not the volume voxel orientation distribution. To exclude non-fibre material, such as matrix and air, an intensity threshold is used to filter out non-fibre voxels. The threshold value is determined using Otsu's threshold, which gives a threshold value of 30.463 for UD-fib and 32.700 for air-tex. Only voxels with values larger than the threshold value are considered to be fibre voxels.

Having filtered out values of $\mathbf{S}$ corresponding to non-fibre voxel positions, the dominant orientation $\mathbf{v}_{1}$ is computed for all remaining (fibre) voxels. This orientation can be interpreted as the orientation of a small piece of a fibre with the size of a single voxel. The orientations of all the fibre voxels are the fibre orientation distribution. At this point, $\theta_{x}, \theta_{x z}$ and $\theta_{x y}$ values for each fibre voxel can be calculated. $\theta_{x}$ is calculated as the absolute angle between $\mathbf{v}_{1}$ and the x-axis. $\theta_{x z}$ is calculated by projecting $\mathbf{v}_{1}$ onto the xz-plane and taking the angle between the projection and the x-axis. $\theta_{x y}$ is calculated by projecting $\mathbf{v}_{1}$ onto the xy-plane and taking the angle between the projection and the $\mathrm{x}$-axis.

The ST method described above has a processing time of less than 10 minutes for each of the tomographic volumes analysed in this study.

\subsection{Comparative verification}

The results using the PCA approach (cf. Figure 14) are compared with the equivalent inclination distribution estimations obtained using the ST method (see Figure 15).

FIGURE 15 
The inclination distributions obtained using the ST method show a great resemblance with the results of the PCA method developed in this study (see Figure 15). No significant deviations are observed between the two methods and since the methods are inherently different the resemblance of the results indicate a corroboration of both methods. A similar comparison was conducted using the statistical tool, KolmogorovSmirnov in [27]. However, due to the large difference in the number of data points of the PCA and ST this would require resampling as described in [28]. Hence, this was considered beyond the scope of this study. However, the data are publicly available [12] in order for this to be conducted in future studies.

In the current case with both high attenuation contrast and high resolution, the fibre tracking approach is considered superior to the thresholding approach because the tracking approach gives additional information, i.e., number of fibres and fibre lengths. However, it is be noted that the ST method has a statistical advantage while the PCA method has the advantage of a straightforward physical interpretation.

\section{Conclusion}

Continuous fibre reinforced composites are increasingly used for structural applications demanding a high stiffness to weight ratio. However, in-depth knowledge of the relation between the, often complex, fibre architecture and macroscopic properties is still lacking. Many recent studies have used X-ray computed tomography (XCT) to characterize the internal three-dimensional microstructure of composite materials. In this study, a novel semi-automatic segmentation procedure is presented. The segmentation algorithm uses an existing fibre tracking algorithm and a novel algorithm using principal component analysis (PCA) to obtain individual fibre inclinations from XCT data. The present approach using PCA is to the knowledge of the authors not found anywhere in literature. The focus of the current segmentation approach is on the single fibre level rather than on the bundle level and is therefore suitable for complex random fibre architectures.

The PCA segmentation algorithm was used to characterize two mutually distinct composites systems, namely a unidirectional glass fibre reinforced thermoset composite (UD-fib) and an air textured glass fibre reinforced composite (air-tex).

The novel segmentation approach resolves the fibre undulation by discretizing the tomographic volume into finite volumes along the length direction of the continuous fibres, i.e., the x-axis. A normalized dimensionless segmentation mesh size of $\Delta \mathrm{x} / D_{f}=10$ was found to be suitable for both material systems. 
The segmentation algorithm was used to obtain the inclination distribution for both material systems. The results show a weighted mean inclination of $2.1^{\circ}$ and $8.0^{\circ}$ as well as the corresponding set of quartiles $[1.4,1.9,2.6,11.3]^{\circ}$ and $[3.7,5.9,10.0,55,7]^{\circ}$ for the UD-fib and air-tex sample, respectively. These results are well within the expected inclination angles for the two material systems. The inclination results for the air-tex sample clearly show how the PCA segmentation approach is capable of resolving high inclination fibre undulation, which was the major goal of the study.

The segmentation approach using PCA was verified by comparison with equivalent inclination distribution results obtained using the structure tensor (ST) method. The qualitative comparative analysis of the two methods showed no significant deviations, i.e., the methods are mutually verified.

The main advantages of the suggested PCA segmentation approach and the individual fibre inclination results obtained can be summarized as:

a) Semi-automated segmentation approach with physical based user input, allowing accurate and repeatable segmentation results.

b) The full segmentation procedure is presented, i.e., fibre detection, fibre tracking and fibre inclination estimation.

c) The novel inclination estimation approach using PCA is verified by comparison with the structure tensor method.

d) The interactive user input is chosen from physical considerations, i.e., the normalized segmentation mesh size $\Delta \mathrm{x} / D_{f}$.

e) Resolving individual fibre undulation of complex fibre architectures.

f) The quantified inclination results serve as a benchmark dataset for future segmentation research because both the raw XCT data and the corresponding inclination results are available for download in $[12]$.

The PCA approach could possibly include quantification of the fibre undulation by considering the ratio between the $1^{\text {st }}$ and $2^{\text {nd }}$ singular value. This could be of interest in future studies.

\section{Data availability}

The raw XCT data and the corresponding inclination segmentation results produced in this study are available for download in [12]. 


\section{CRediT authorship contribution statement}

Filip Bo Salling: Conceptualization, Methodology, Software, Formal Analysis, Investigation, Visualization, Writing - Original Draft, Writing - Review \& Editing. Niels Jeppesen: Software (Sec. 6), methodology (Sec. 6), Writing - Original Draft (section 6.1), Writing - Review \& Editing. Mads R. Sonne: Supervision. Jesper H. Hattel: Funding acquisition, Writing - Review \& Editing. Lars P. Mikkelsen: Conceptualization, Methodology, Supervision, Writing - Review \& Editing.

\section{Declaration of competing interest}

The authors declare that they have no known competing financial interests or personal relationships which have or could be perceived to have influenced the work reported in this article.

\section{Acknowledgements}

This work is part of the Resin Injection Pultrusion (RIP) project which has been granted by the Danish Council for Independent Research | Technology and Production Sciences (DFF/FTP), Grant no. DFF-6111-00112.

\section{References}

[1] Markets and Markets, Lakshmi Narayanan, July 2020, Composites Market by Fibre Type (Glass Fibre Composites, Carbon Fibre Composites, Natural Fibre Composites), Resin Type (Thermoset Composites, Thermoplastic Composites), manufacturing process, End-use Industri and Region - Global Forecast to 2025 https://www.marketsandmarkets.com/MarketReports/composite-market-200051282.html, Visited 05-11-2020.

[2] Mikkelsen LP, Emerson MJ, Jespersen KM, Dahl VA, Conradsen K, Dahl AB 2016. X-ray based micromechanical finite element modeling of composites materials. Proc. Of $29^{\text {th }}$ Nordic Seminar on Computational Mechanics.

[3] Mikkelsen LP 2020. The fatigue damage evalution in the load carrying composites laminates of 
wind turbine blades. Chp. 16 in: Fatigue Life Prediction of Composites and Composite Structures (2 ${ }^{\text {nd }}$ ed.), Woodhead Publishing series in Compos. Sci. and Eng., p 569-603.

[4] Agarwal BD, Broutman LJ 1990. Analysis and performance of fibre composites $4^{\text {th }}$ ed. (Hoboken/NJ, USA: Wiley).

[5] Paluch B 1996. Analysis of geometric imperfections affecting the fibers in unidirectional composites. Jour. of Composite Materials. 30:454-85.

[6] Clarke AR, Archenhold G, Davidson NC 1995. A novel technique for determining the 3d spatial distribution of glass fibres in polymer composites. Composites Science and Technology. 55:7591.

[7] Baran I, Straumit I, Shishkina O, Lomov SV 2018. X-ray computed tomography characterization of manufacturing induced defects in a glass/polyester pultruded profile. Composite Structures. 195:74-82.

[8] Klingaa CG, Dahmen T, Baier S, Mohanty S, Hattel JH 2020. X-ray CT and image analysis methodology for local roughness characterization in cooling channels made by metal additive manufacturing. Additive Manufacturing. 32.

[9] Ren W, Yang Z, Sharma R, Zhang C, Withers PJ 2015. Two-dimensional X-ray CT image based meso-scale fracture modelling of concrete. Engineering Fracture Mechanics. 133:24-39.

[10] Emerson MJ, Dahl VA, Conradsen K, Mikkelsen LP, Dahl AB 2017. Statistical validation of individual fibre segmentation from tomograms and microscopy. Composites Science and Technology. 160:208-15.

[11] Garcea SC, Wang Y, Withers PJ 2018. X-ray computed tomography of polymer composites. Composites Science and Technology. 156:305-19.

[12] Salling, Filip Bo, Hattel, Jesper H., \& Mikkelsen, Lars P. (2021). X-ray computed tomography and scanning electron microscopy datasets of unidirectional and textured glass fibre composites. [Data set]. Zenodo. https://doi.org/10.5281/zenodo.5483719

[13] Wilhelmsson D, Mikkelsen LP, Fæster S, Asp LE 2019. Influence of in-plane shear on kink-plane orientation in a unidirectional fibre composite. Composites Part A: App. Sci. and Manufacturing. 119:283-90.

[14] Wilhelmsson D, Mikkelsen LP, Fæster S, Asp LE 2019. X-ray tomography data of compression 
tested unidirectional fibre composites with different off-axis angles. Data in Brief. $\mathbf{2 5}$

[15] Varandas LF, Catalanotti G, Melro AR, Tavares RP, Falzon BG 2020. Micromechanical modelling of the longitudinal compressive and tensile failure of unidirectional composites: The effect of gibre misalignment introduced via a stochastic process. Int. Journ. Of Solids and Structures. 203:157-76.

[16] Auenhammer RM, Mikkelsen LP, Asp LE, Blizler BJ 2020. Automated X-ray computer tomography segmentation method for finite element analysis of non-crimp fabric reinforced composites. Composite Structures, 256.

[17] Auenhammer RM, Mikkelsen LP, Asp LE, Blinzler BJ 2020. X-ray tomography based numerical analysis of stress concentration in non-crimp fabric reinforced composites - assesment of segmentation methods. IOP Conference Serie: Materials Science and Engineering. 942 [012038].

[18] Advani SG, Tucker CL 1987. The use of tensors to describe and predict fiber orientation in short fiber composites. Jour. of Rheology. 31(8):751-84.

[19] Jeppesen N, Dahl VA, Christensen AN, Dahl AB, Mikkelsen LP 2020. Characterization of the fiber orientations in non-crimp glass fiber reinforced composites using structure tensor. IOP Conference Serie: Materials Science and Engineering, 942, [012037].

[20] Shkarin R, Shkarin A, Shkarin S, Agelica C, Surmenev RA, Surmeneva MA, Weinhardt V, Baumbach T, Mikut R 2019. Quanfima: An open source Python package for automated fiber analysis of biomaterials. Plos One, Vol. 14 Issue 4, pp. e0215137.

[21] Emerson MJ, Jespersen KM, Dahl AB, Conradsen K, Mikkelsen LP 2017. Individual fiber segmentation from 3D x-ray computed tomography for characterising the fibre orientation in unidirectional composite materials. Compos. part A: App. Sci. and Manuf. 97:83-92.

[22] Rasmussen FS, Emerson MJ, Sonne MR, Hattel JH, Mikkelsen LP and Dahl VA 2019. Fiber segmentation from 3D X-ray computed tomography of composites with continuous and textured glass fiber yarns. Proc. of 2019 Int. Conf. on Tomography of Mater. \& Struct. (Cairns, Australia).

[23] Amrehn M, Steidl S Kortekaas R, Strumia M, Weingarten M, Kowarschik M, Maier A 2019. A semi-automated usability evaluation framework for interactive image segmentation systems. Int. Jour. Of Biomedical Imaging. 
[24] Jeppesen, N., Mikkelsen, L.P., Dahl, A.B., Christensen, A.N., Dahl, V.A., Quantifying effects of manufacturing methods on fiber orientation in unidirectional composites using structure tensor analysis, Composites Part A, 149, 106541, https://doi.org/10.1016/j.compositesa.2021.106541, 2021.

[25] Serafin J, Olson E, Grisetti G 2016. Fast and robust 3D feature extraction from sparse point clouds. IEE/RSJ Int. Conf. on Intelligent Robots and Systems (IROS), Daejeon (South Korea), pp. 410512.

[26] Krenchel H 1964. Fiber reinforcement: Theoretical and practical investigations of the elasticity and strength offiber-reinforced materials. Akademisk forlag, Danmarks tekniske højskole.

[27] Rosini S, Mavrogordato MN, Egorova O, Matthews ES, Jackson SE, Spearing SM and Sinclair I 2019. In situ statistical measurement of local morphology in carbon-epoxy composites using synchrotron X-ray computed tomography. Composites Part A, 125.

[28] Breite C 2021. Aligning fibre break models for composites with the observable micro-scale material behaviour. PhD Dissertation - KU Leuven, Science, Engineering \& Technology.
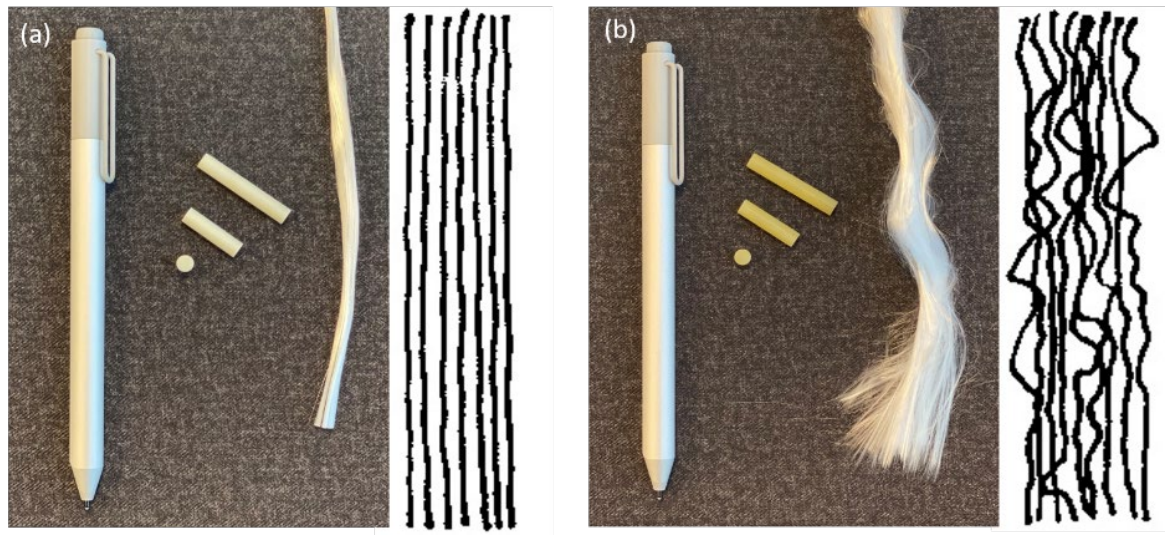

Figure 1. Pictures of composite samples and their respective fibre reinforcement:

a) UD-fib, b) air-tex. 

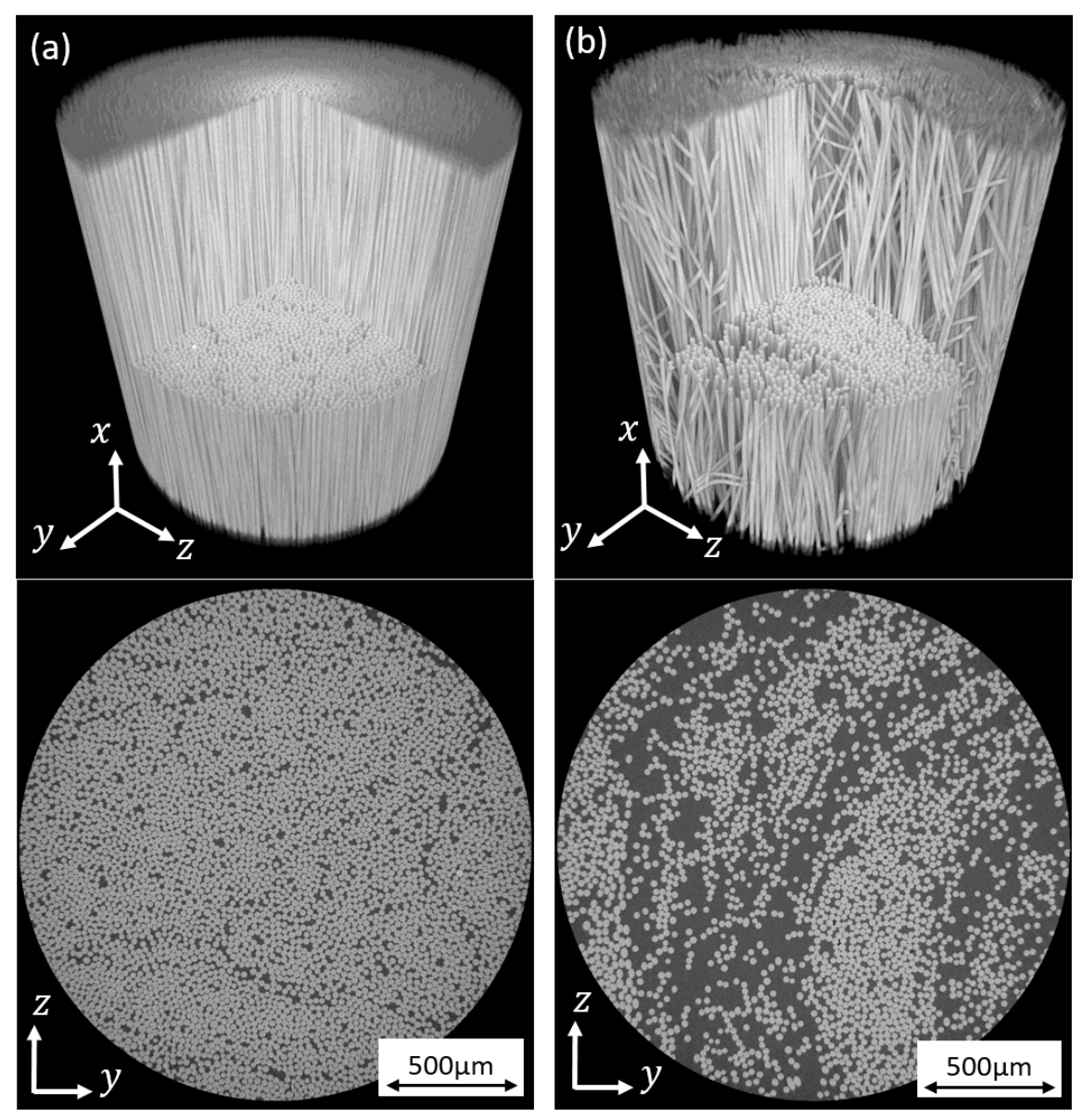

Figure 2. 3D tomograms of $988 \times 1013 \times 999$ voxels with a voxel size of $1.99 \mu \mathrm{m}$ together with a corresponding 2D cross-sectional image slice in the yz-plane: a) UD-fib, b) air-tex.
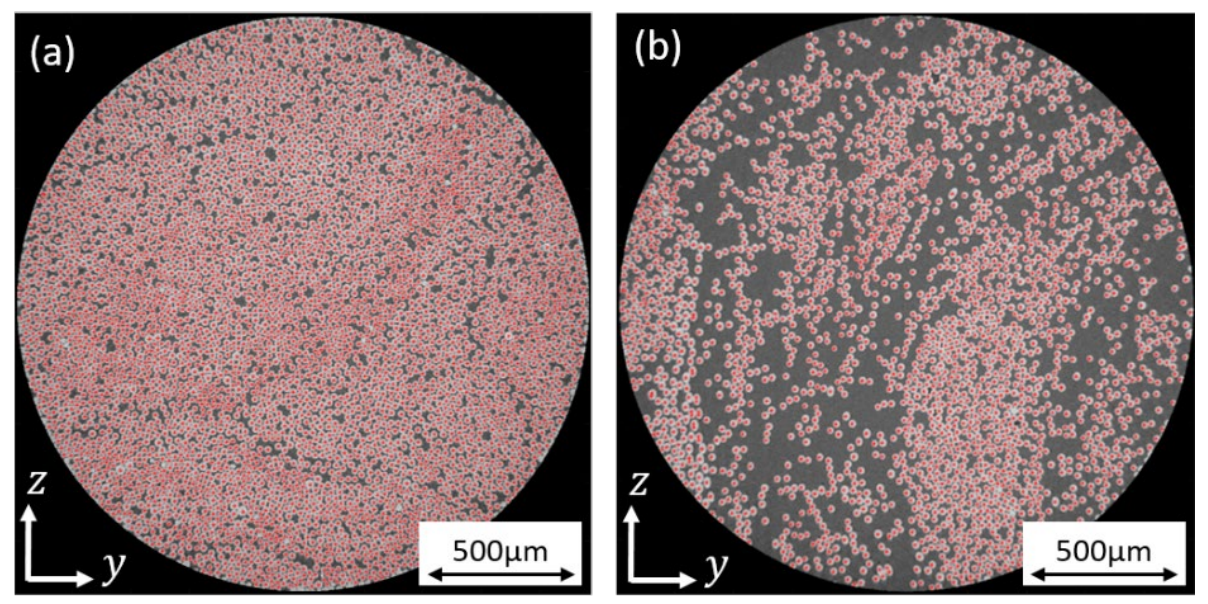

Figure 3. 2D image slices from $\mathrm{XCT}$ with fibre centre detections:

a) UD-fib, b) air-tex. 

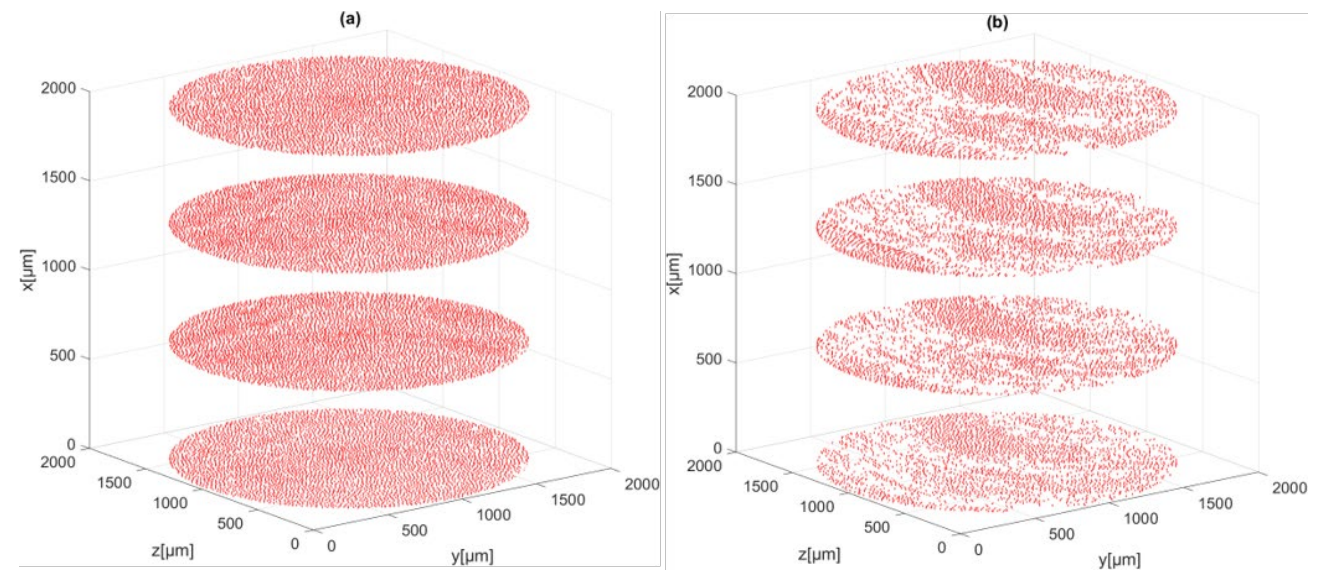

Figure 4. Four evenly spaced stacks of five image slices with detected fibre centres:

a) UD-fib, b) air-tex. 

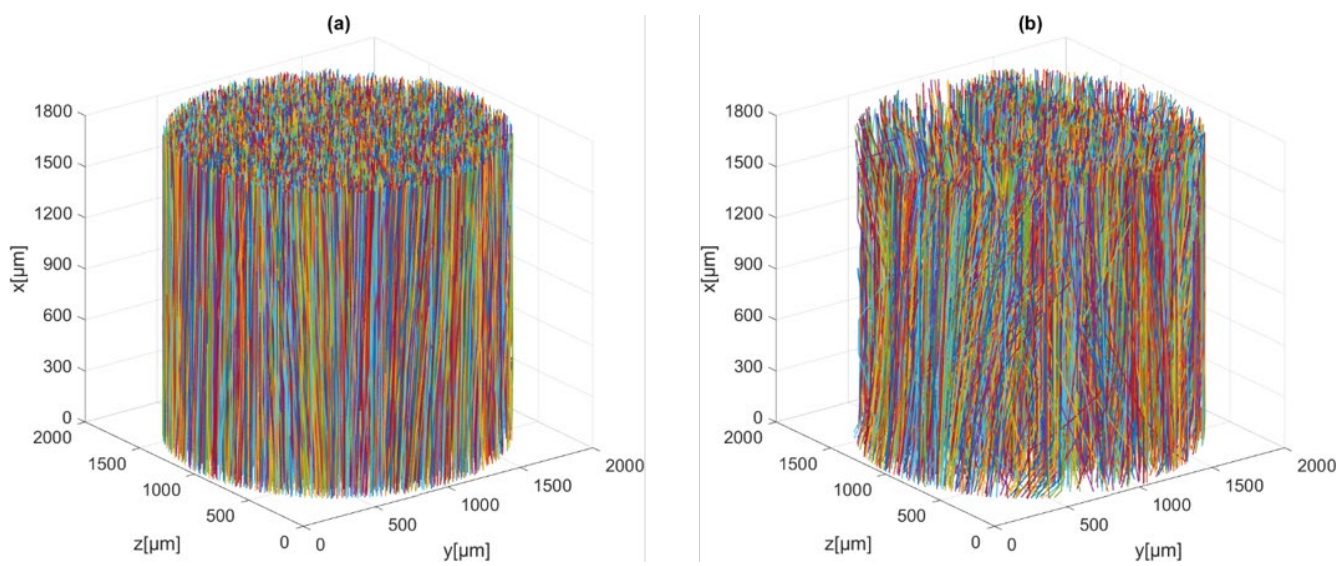

Figure 5. All the 3D fibre trajectories obtained by the fibre tracking segmentation algorithm:

a) UD-fib, b) air-tex.
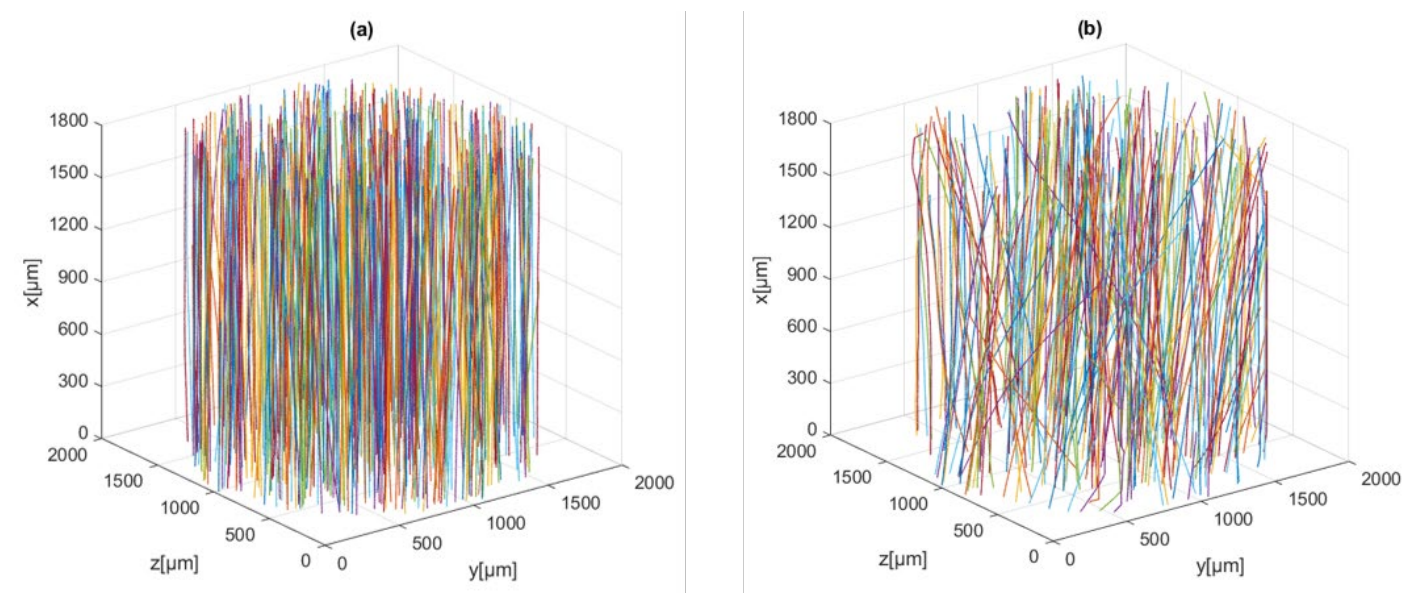

Figure 6. $10 \%$ of the 3D fibre trajectories obtained by the fibre tracking algorithm:

a) UD-fib, b) air-tex. 
(a)

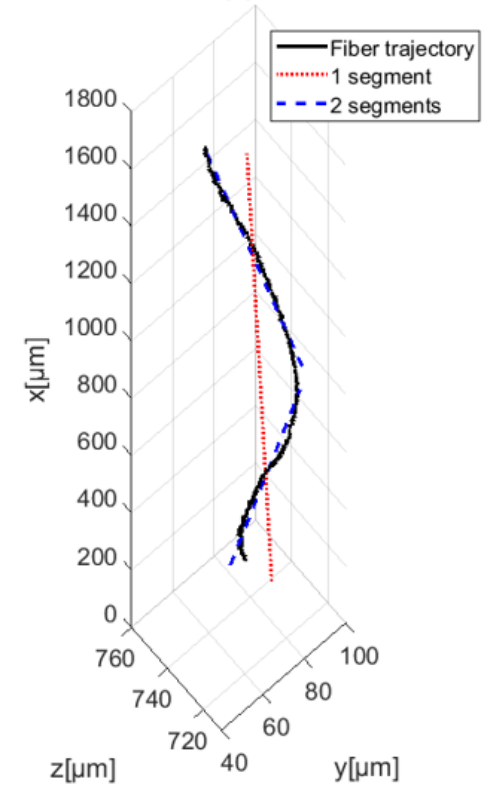

(b)

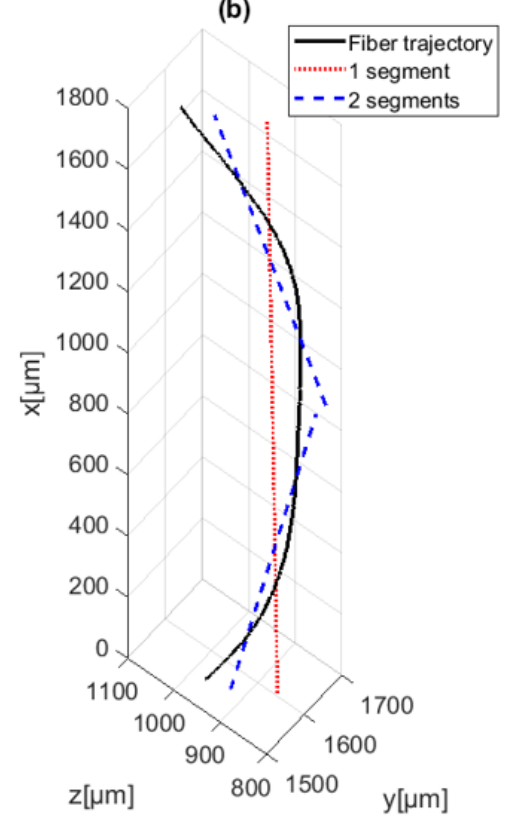

Figure 7. Example of a fibre trajectory and the corresponding linear representation by 1 or 2 segments: a) UD-fib, b) air-tex.

(a)

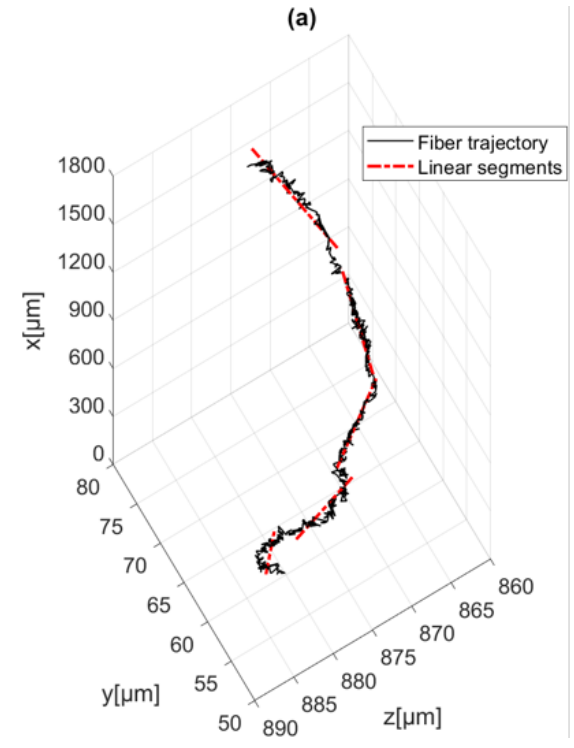

(b)

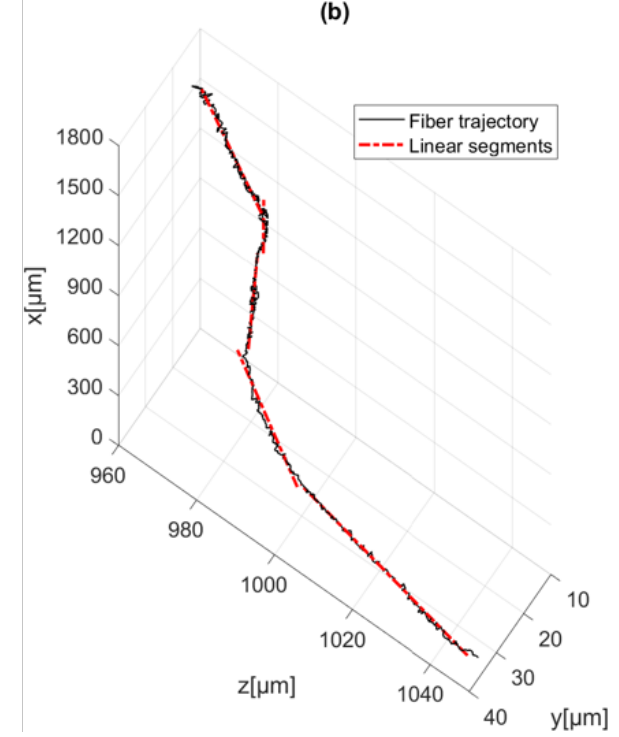

Figure 8. Example of a fibre trajectory and the corresponding linear representation by five fibre subsegments with a dimensionless height of $\Delta \mathrm{x} / D_{f} \approx 15$ (to be explained):

a) UD-fib, b) air-tex. 


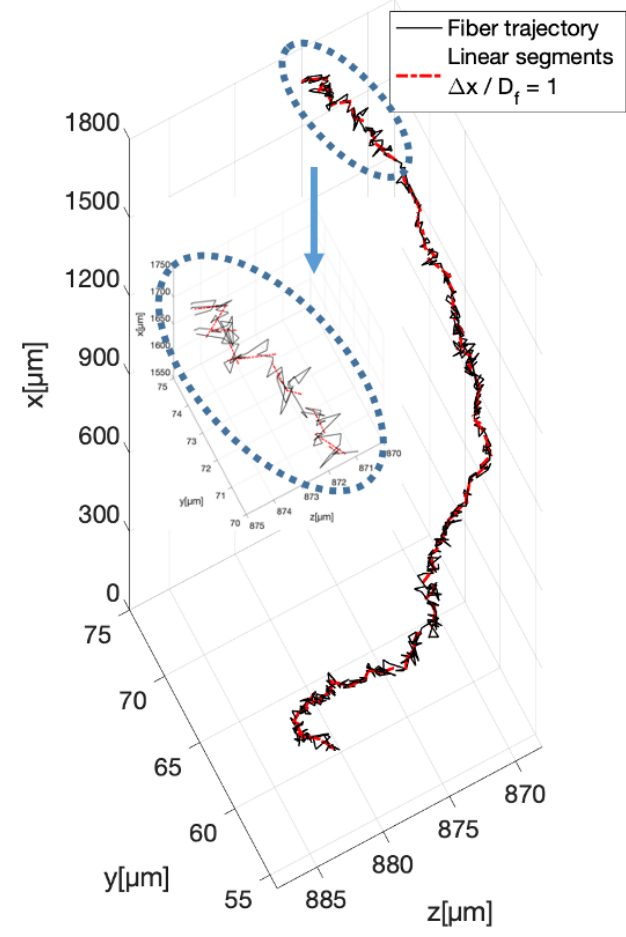

Figure 9. Fibre trajectory and corresponding linear representation using a segmentation mesh with

$$
\Delta \mathrm{x} / D_{f} \approx 1, \mathrm{UD} \text {-fib. }
$$

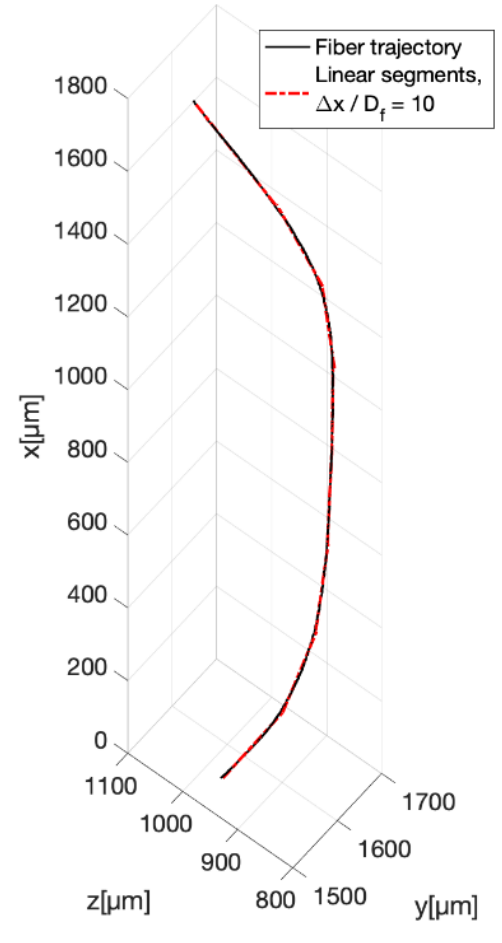

Figure 10. Fibre trajectory and corresponding linear representation using a segmentation mesh with $\Delta \mathrm{x} / D_{f} \approx 10$, air-

tex.

Table 1. Overview of the differences between different dimensionless segmentation mesh sizes.

\begin{tabular}{l|c|c|c|c}
\hline \# fibre sub-segments & 1 & 5 & 8 & 81 \\
\hline \# image slices / fibre sub-segment & 900 & 180 & 113 & 12 \\
\hline $\boldsymbol{\Delta x / \boldsymbol { D } _ { \boldsymbol { f } }}$ & 77 & 15 & 10 & 1 \\
\hline Figure ref. & Fig. 7 & Fig. 8 & Fig. 10 & Fig. 9 \\
\hline
\end{tabular}


Table 2. Summary of the three individual fibre segmentation steps.

\begin{tabular}{|c|c|c|c|}
\hline Segmentation step & $\begin{array}{l}\text { Segmentation } \\
\text { algorithm }\end{array}$ & User input & $\begin{array}{l}\text { Processing } \\
\text { time }\end{array}$ \\
\hline i) Fibre centre detections & {$[21]$} & $\begin{array}{l}\text { RoI: the full } 988 \times 1013 \times 999 \\
\text { voxels volume } \\
\text { Patch size: } 11 \text { pixels } \\
\text { Threshold: } 0.5\end{array}$ & $\sim 1 \mathrm{~h}$ \\
\hline ii) Fibre tracking & [21] & $\begin{array}{l}\text { Image slices: } 51-950 \\
\Delta C_{\max }{ }^{*}=6 \text { pixels } \\
\end{array}$ & $<5 \min$ \\
\hline iii) Inclination estimation & PCA & $\begin{array}{l}\frac{\Delta x}{D_{f}}=[77: 1] \\
\left\{\frac{\Delta x}{D_{f}}\right\}_{o p t}=10 \\
\min \left(N_{C}\right)^{* *} \geq 95 \%\end{array}$ & $<15 \mathrm{~s}$ \\
\hline
\end{tabular}

* Maximum allowed fibre centre movement from one slice to the next.

${ }^{* *}$ Minimum fraction of fibre centres detected to accept PCA.
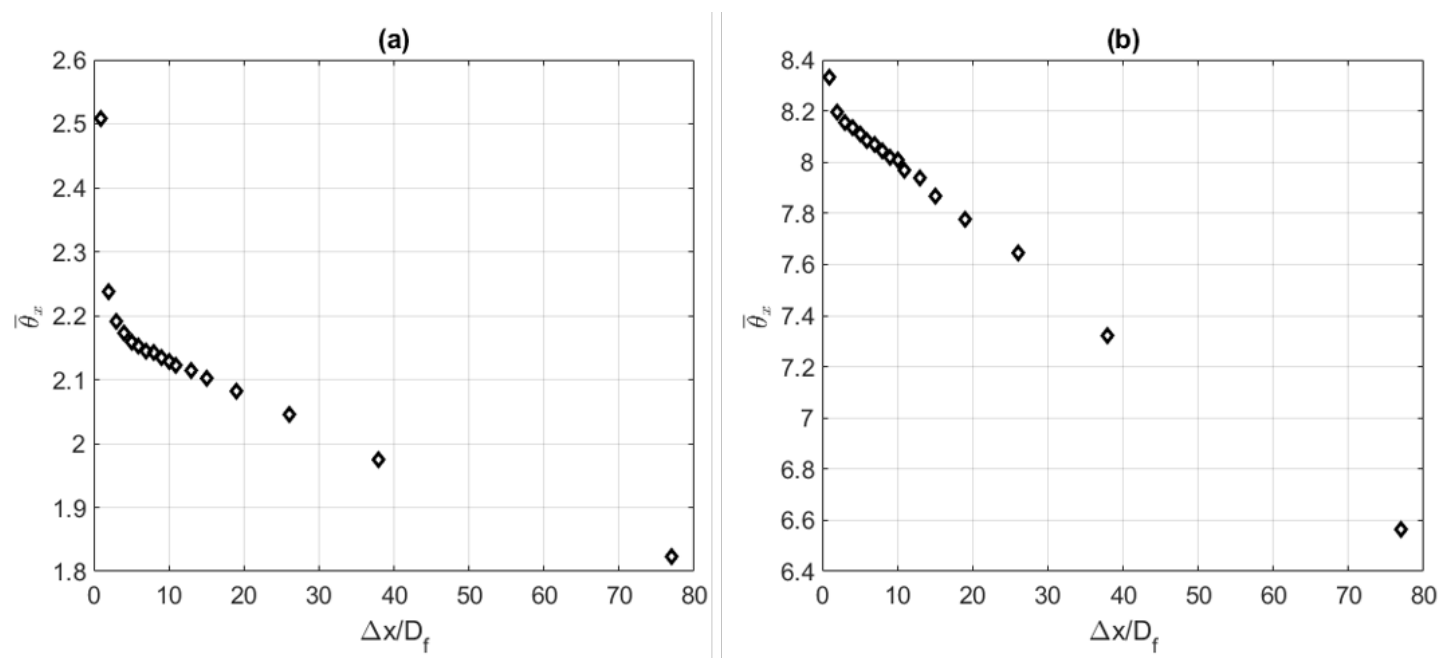

Figure 11. Weighted mean inclination as a function of dimensionless segmentation mess:

a) UD-fib, b) air-tex. 
(a)
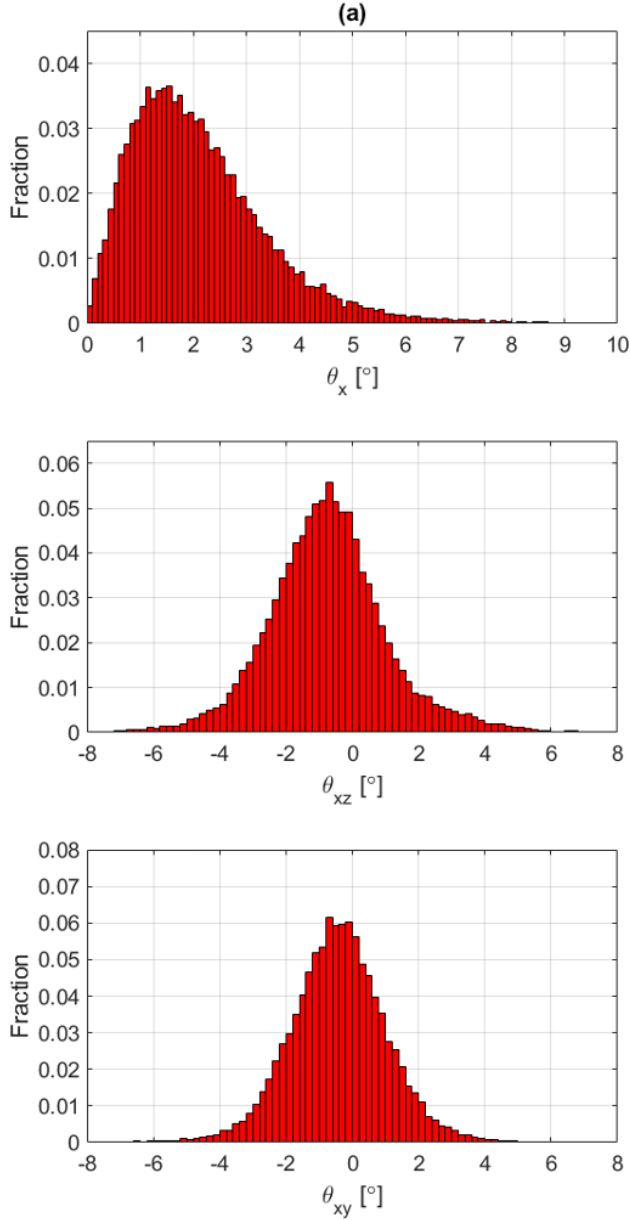

(b)
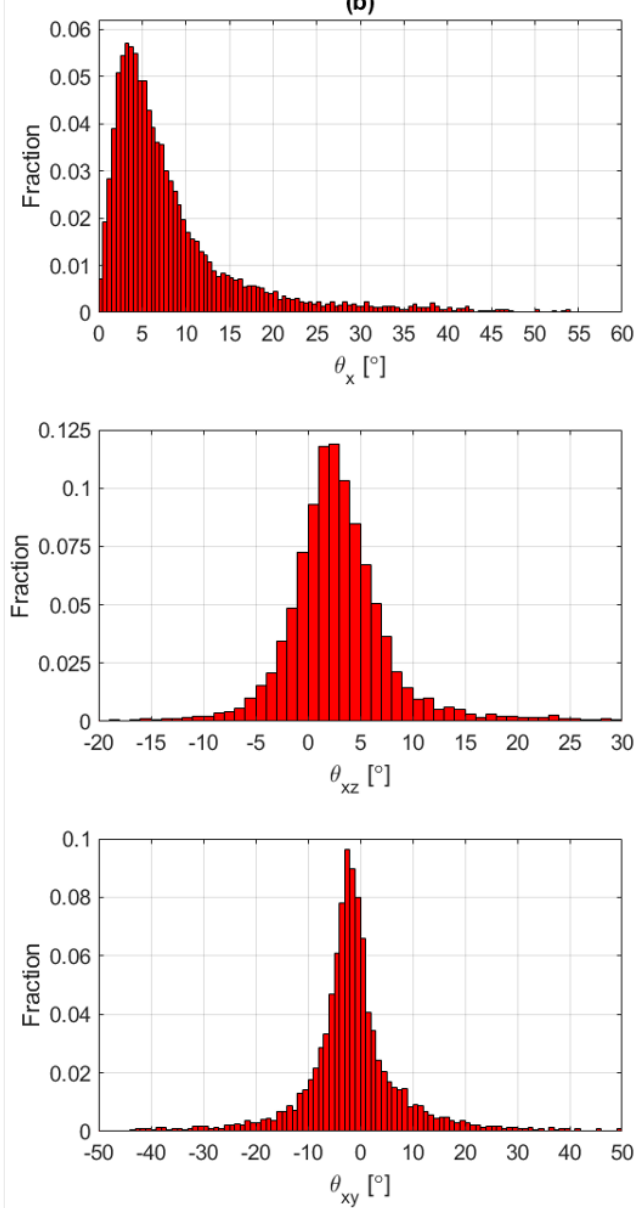

Figure 12. Inclination distribution and projected inclination distributions obtained with a segmentation mesh size of $\frac{\Delta x}{D_{f}}=10$ : a) UD-fib, b) air-tex. 
Table 3. Weighted mean and quartiles of the inclination distribution and weighted mean projection inclinations (cf. Figure 12).

\begin{tabular}{l|c|c|c|c|c|c|c}
\hline Material System & $\bar{\theta}_{x}$ & $\bar{\theta}_{x y}$ & $\bar{\theta}_{x z}$ & $Q_{x 1}$ & $Q_{x 2}$ & $Q_{x 3}$ & $Q_{x 4}$ \\
\hline (a) UD-fib & $2.1^{\circ}$ & $-0.7^{\circ}$ & $-0.4^{\circ}$ & $1.4^{\circ}$ & $1.9^{\circ}$ & $2.6^{\circ}$ & $11.3^{\circ}$ \\
\hline (b) Air-tex & $8.0^{\circ}$ & $-1.5^{\circ}$ & $2.6^{\circ}$ & $3.7^{\circ}$ & $5.9^{\circ}$ & $10.0^{\circ}$ & $55.7^{\circ}$ \\
\hline
\end{tabular}
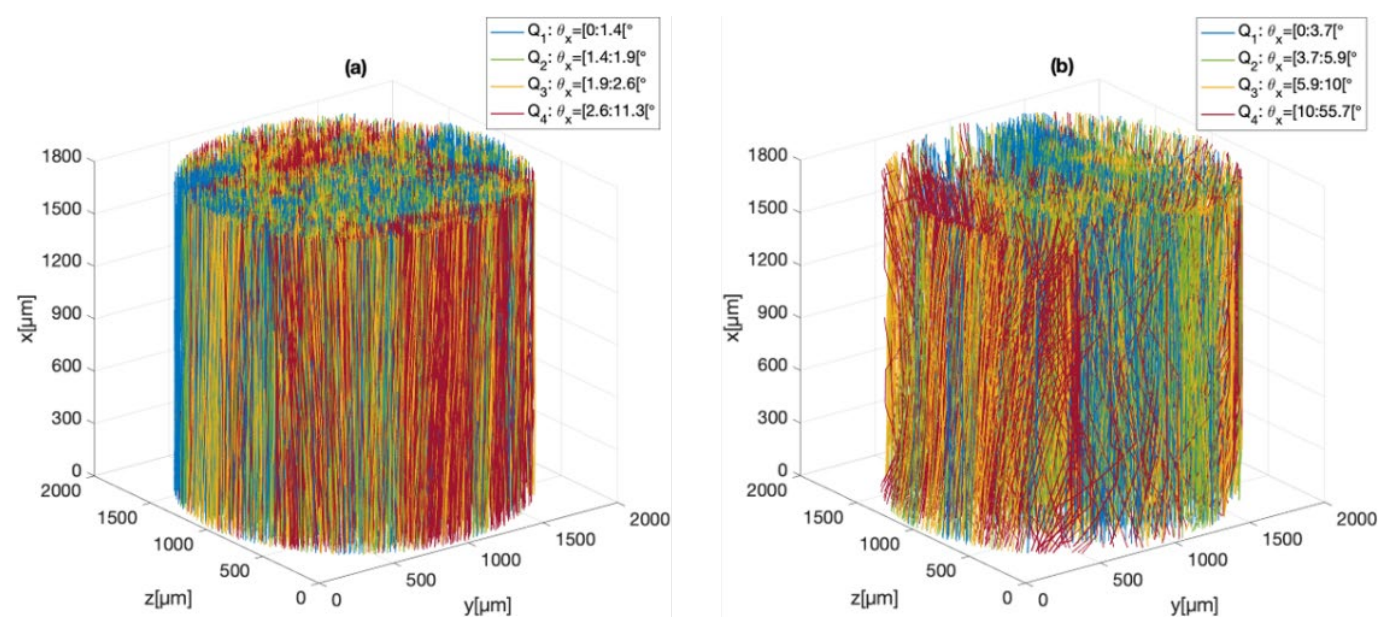

Figure 13. 3D volume of fibre trajectories coloured according to their individual mean inclination using the quartiles as interval boundaries: a) UD-fib, b) air-tex.
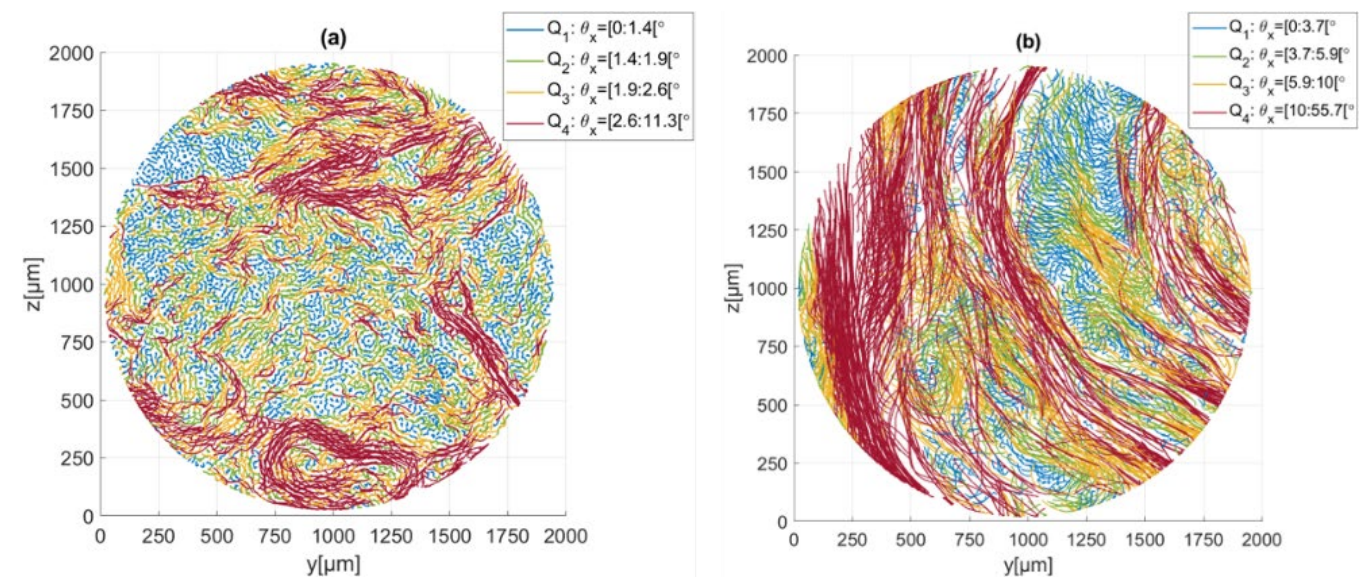

Figure 14. Cross-sectional view of fibre trajectories coloured according to their individual mean inclination using the quartiles as interval boundaries: a) UD-fib, b) air-tex. 
(a)

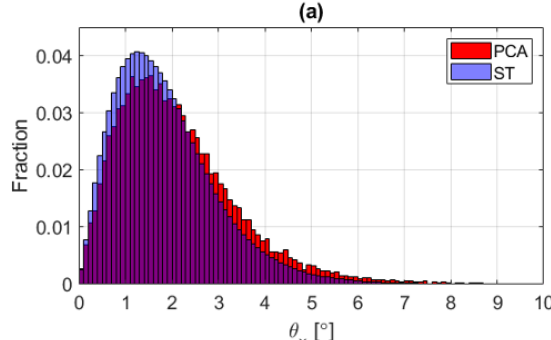

$\theta_{\mathrm{x}}\left[{ }^{\circ}\right]$
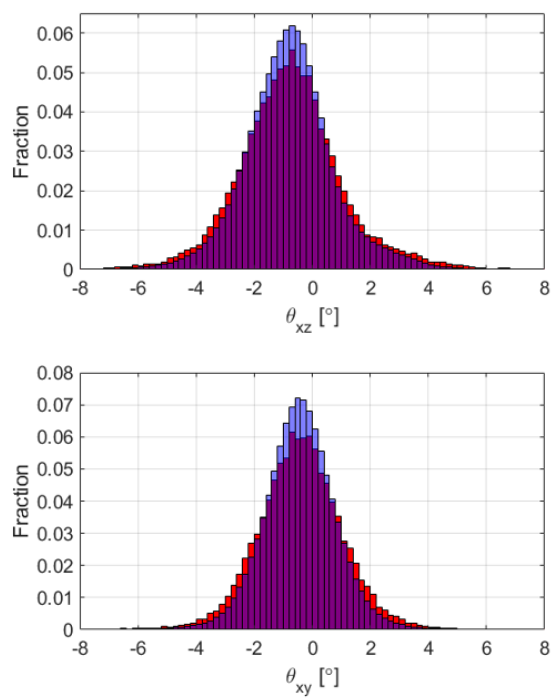

(b)
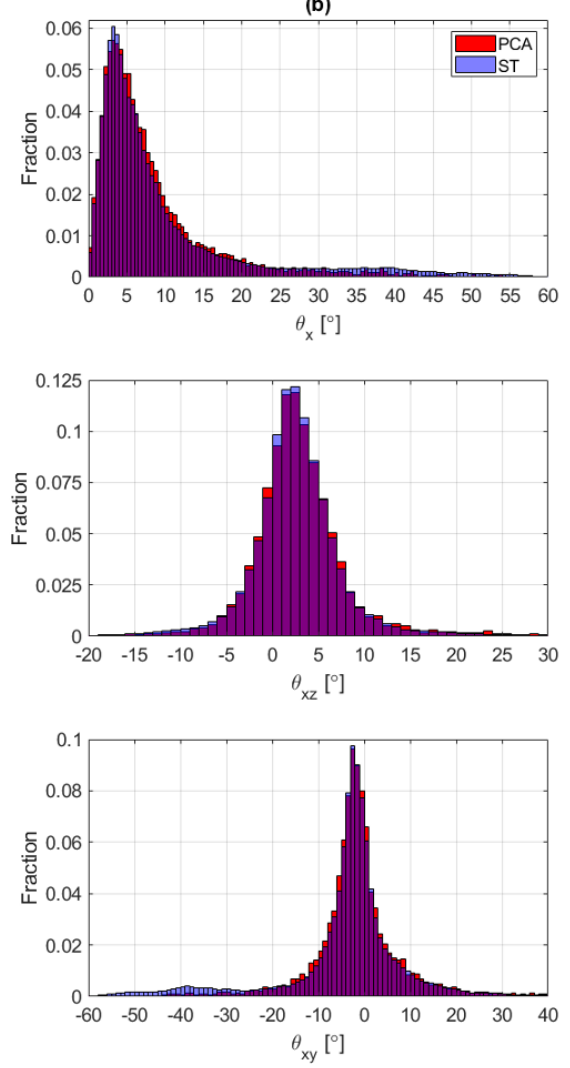

Figure 15. Comparison of the inclination distributions obtained using the PCA method (red) and the ST method (blue): a) UD-fib, b) air-tex. 